doi: https://doi.org/10.15407/dopovidi2018.04.041

УДК 517.36

\title{
А.C. Хорошун
}

Институт механики им. С.П. Тимошенко НАН Украины, Киев

E-mail: khoroshunanatoliy@gmail.com

\section{О построении управления движением маятника вращением инерциального маховика}

Представлено академиком НАН Украины А.А. Мартынюком

Рассмотрен пример малоприводной механической системь, которая предоставляет собой маятник, управление движением которого происходит благодаря вращению маховика. Получен в явном виде закон управления вращением маховика, обеспечивающего стабилизащию верхнего положения равновесия маятника.

Ключевые слова: малоприводная механическая система, глобальная асимптотическая устойчивость, инерииальный маховик, положение равновесия.

Малоприводные механические системы (MMC) характеризуются тем, что количество управляющих входов в такой системе меньше, чем количество переменных, которые описывают их поведение. Системы этого класса широко используются при конструировании различных роботов, аэрокосмических и морских аппаратов (см. [1]), поскольку имеют преимущество в меньшем потреблении энергии и меньшую стоимость.

В работе [2] рассмотрен пример ММС и получен закон управления, обеспечивающий глобальную асимптотическую устойчивость состояния равновесия этой системы, а также дана оценка области в пространстве параметров модели, при всех значениях параметров из которой построенное управление также реализует поставленную задачу.

В данной статье рассмотрен маятник, управление движением которого происходит с помощью вращения маховика. Эта модель ММС была впервые предложена к рассмотрению автором статьи [3]. В работах [3-6] используются различные подходы к построению управления движением маятника. Закон управления, который обеспечит стабилизацию верхнего положения равновесия маятника, полученный в работе [2], будет представлен в явном виде, т. е. зависящим от физических характеристик модели (скорости, смещений, времени), что важно для его практической реализации.

C) A.C. Хорошун, 2018 


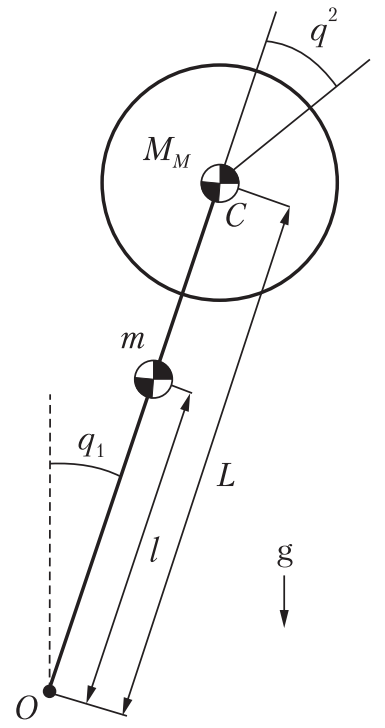

Puc. 1

Основной результат. Описание устройства ММС маятник с маховиком (англ. Inverted Wheel Pendulum) дано в работе [2]. На рис. 1 изображена схема маятника с маховиком, где $O$ - точка подвеса маятника, $C$ - центр маховика, $q_{1}-$ угол поворота маятника относительно вертикали, $q_{2}$ - угол поворота маховика относительно маятника.

Уравнения движения рассматриваемой модели имеют следующий вид:

$$
\left\{\begin{array}{l}
J_{1}(p) \ddot{q}_{1}+J_{2}(p) \ddot{q}_{2}-\omega(p) \sin \left(q_{1}\right)=0 \\
J_{2}(p) \ddot{q}_{1}+J_{2}(p) \ddot{q}_{2}=\Delta
\end{array}\right.
$$

где $J_{1}(p)=J_{m}(p)+\left(M_{M}(p)+M_{R}(p)\right) L^{2}(p)+J_{2}(p), J_{2}(p)=J_{M}(p)+$ $+J_{R}(p), \omega(p)=\left(m(p) l(p)+\left(M_{M}(p)+M_{R}(p)\right) L(p)\right) g, g-$ ускорение свободного падения; $M_{M}(p)$ - масса маховика; $m(p)-$ масса маятника; $M_{R}(p)$ - масса электродвигателя; $J_{m}(p), J_{M}(p)$ и $J_{R}(p)$ - моменты инерции маятника, маховика и ротора двигателя, соответственно, относительно их осей вращения; $L(p)$ - длина маятника; $l(p)$ - расстояние от шарнира до центра масс маятника; $\Delta$ - момент электромагнитных сил, приложенных к ротору электродвигателя со стороны статора; $p \in P \subseteq R^{n}-$ векторный параметр, описывающий возможные неточности модели, $n \in N$.

Задача состоит в том, чтобы выбрать закон управления $\Delta$, который стабилизирует верхнее положение равновесия маятника в то время, как маятник прекратит свое вращение, вне зависимости от начальных значений переменных.

Введя безразмерные переменные $\tau=t \sqrt{\frac{\omega(p)}{J_{2}(p)}}, v=\frac{\Delta}{\omega(p)}$, получим безразмерную систему дифференциальных уравнений, эквивалентную системе дифференциальных уравнений (1):

$$
\left\{\begin{array}{l}
\frac{J_{1}(p)}{J_{2}(p)} \ddot{q}_{1}+\ddot{q}_{2}-\sin \left(q_{1}\right)=0, \\
\ddot{q}_{1}+\ddot{q}_{2}=v .
\end{array}\right.
$$

Здесь дифференцирование ведется по безразмерному времени $\tau$. Пусть $v=\alpha u+\beta$, где $\alpha=1-\frac{J_{2}(p)}{J_{1}(p)}, \beta=\frac{J_{2}(p)}{J_{1}(p)} \sin \left(q_{1}\right)$, тогда заменой переменных

$$
\left\{\begin{array}{l}
\eta_{1}=\frac{J_{1}(p)}{J_{2}(p)} \dot{q}_{1}+\dot{q}_{2} \\
\eta_{2}=q_{1} \\
\eta_{3}=\dot{q}_{2}
\end{array}\right.
$$


система дифференциальных уравнений (2) приводится к “каскадному” виду

$$
\left\{\begin{array}{l}
\dot{\eta}_{1}=\sin \left(\eta_{2}\right) \\
\dot{\eta}_{2}=\frac{J_{2}(p)}{J_{1}(p)}\left(\eta_{1}-\eta_{3}\right) \\
\dot{\eta}_{3}=u
\end{array}\right.
$$

из глобальной устойчивости состояния равновесия которой при управлении $u$ следует аналогичное свойство системы дифференциальных уравнений (2) при управлении $v$.

Применяя так называемый Dynamic Surface Control, см. [2, 7], получим, что управление $u=-K_{2} x_{3}-\frac{y}{\tau_{3}}$, где переменные $\left(\eta_{1}, \eta_{2}, \eta_{3}\right)^{T}$ и $\left(x_{1}, x_{2}, x_{3}, x_{4}, y\right)^{T}$ связаны соотношениями

$$
\left\{\begin{array}{l}
\eta_{1}=x_{1}, \\
\eta_{2}=x_{2}+x_{4}-\arctan \left(x_{1}\right), \\
\eta_{3}=x_{3}+y+\frac{J_{1}(p)}{J_{2}(p)}\left(K_{1} x_{2}+\frac{J_{2}(p)}{J_{1}(p)} x_{1}+\frac{x_{4}}{\tau_{2}}\right)
\end{array}\right.
$$

стабилизирует состояние равновесия системы дифференциальных уравнений (4) вне зависимости от начальных значений переменных, если нулевое состояние равновесия системы дифференциальных уравнений:

$$
\left\{\begin{array}{l}
\dot{x}_{1}=\sin \left(x_{2}+x_{4}-\arctan \left(x_{1}\right)\right) \\
\dot{x}_{2}=-K_{1} x_{2}-\frac{J_{2}(p)}{J_{1}(p)} x_{3}-\frac{J_{2}(p)}{J_{1}(p)} y, \\
\dot{x}_{3}=-K_{2} x_{3} \\
\dot{x}_{4}=-\frac{x_{4}}{\tau_{2}}+\frac{\sin \left(x_{2}+x_{4}-\arctan \left(x_{1}\right)\right)}{1+\left(x_{1}\right)^{2}} \\
\dot{y}=-\frac{y}{\tau_{3}}+F\left(p, x_{1}, x_{2}, x_{3}, x_{4}, y\right)
\end{array}\right.
$$

где

$$
\begin{aligned}
& F\left(p, x_{1}, x_{2}, x_{3}, x_{4}, y\right)=\frac{J_{1}(p)}{J_{2}(p)} K_{1}^{2} x_{2}+K_{1} x_{3}+K_{1} y-\sin \left(x_{2}+x_{4}-\arctan \left(x_{1}\right)\right)+ \\
& +\frac{J_{1}(p)}{J_{2}(p)} \frac{x_{4}}{\tau_{2}^{2}}-\frac{J_{1}(p)}{J_{2}(p) \tau_{2}} \frac{\sin \left(x_{2}+x_{4}-\arctan \left(x_{1}\right)\right)}{1+\left(x_{1}\right)^{2}}
\end{aligned}
$$

и глобально асимптотически устойчиво. В работе [2] такая устойчивость доказана. приведены способы выбора параметров управления $K_{1}, K_{2}, \tau_{2}, \tau_{3}$ и определения оценок области $P$, при всех значениях параметров из которой, данный тип устойчивости при выбранном управлении сохраняется. 

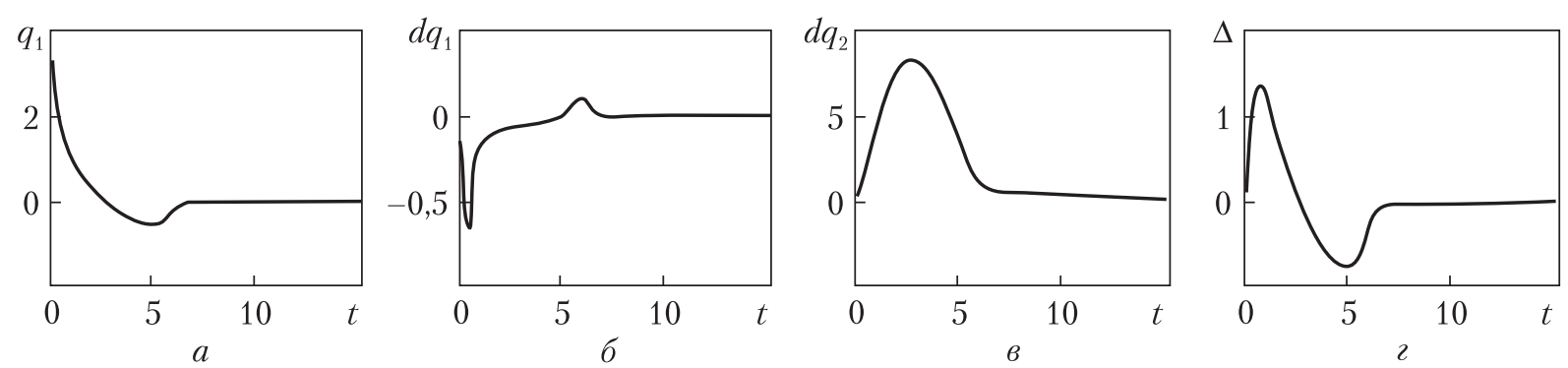

Puc. 2

Пусть $K_{1}$ и $\tau_{2}$ выбраны таким образом, что $K_{1}=\frac{1}{\tau_{2}}$. Тогда из (3) и (5) следует

$$
\begin{aligned}
& x_{2}+x_{4}=\eta_{2}+\arctan \left(\eta_{1}\right)=q_{1}+\arctan \left(\frac{J_{1}(p)}{J_{2}(p)} \dot{q}_{1}+\dot{q}_{2}\right), \\
& x_{3}+y=\eta_{3}-\frac{J_{1}(p)}{J_{2}(p)}\left(\frac{J_{2}(p)}{J_{1}(p)} \eta_{1}+K_{1}\left(x_{2}+x_{4}\right)\right)= \\
& =-\frac{J_{1}(p)}{J_{2}(p)} \dot{q}_{1}-\frac{J_{1}(p)}{J_{2}(p)} K_{1}\left(q_{1}+\arctan \left(\frac{J_{1}(p)}{J_{2}(p)} \dot{q}_{1}+\dot{q}_{2}\right)\right) . \text { Выразив величину } x_{3}(\tau) \text { из (6) в }
\end{aligned}
$$

виде $x_{3}(\tau)=x_{3}(0) \exp \left(-K_{2} \tau\right)$, получим закон управления $u$, который явно зависит от физических характеристик исходной модели, в виде

$$
\begin{aligned}
& u=-K_{2} x_{3}-\frac{y}{\tau_{3}}=\left(-K_{2}+\frac{1}{\tau_{3}}\right) x_{3}-\frac{1}{\tau_{2}}\left(x_{3}+y\right)= \\
& =\left(-K_{2}+\frac{1}{\tau_{3}}\right) x_{3}(0) \exp \left(-K_{2} \tau\right)+\frac{J_{1}(p)}{J_{2}(p) \tau_{3}} \dot{q}_{1}+\frac{J_{1}(p) K_{1}}{J_{2}(p) \tau_{3}}\left(q_{1}+\arctan \left(\frac{J_{1}(p)}{J_{2}(p)} \dot{q}_{1}+\dot{q}_{2}\right)\right)
\end{aligned}
$$

или, переходя к размерным величинам, закон управления $\Delta-$ в виде

$$
\begin{aligned}
& \Delta=\omega(p)\left(1-\frac{J_{2}(p)}{J_{1}(p)}\right)\left[\left(-K_{2}+\frac{1}{\tau_{3}}\right) x_{3}(0) \exp \left(-K_{2} \sqrt{\frac{\omega(p)}{J_{2}(p)}} t\right)+\frac{J_{1}(p)}{J_{2}(p) \tau_{3}} \sqrt{\frac{J_{2}(p)}{\omega(p)}} \dot{q}_{1}+\right. \\
& \left.+\frac{J_{1}(p) K_{1}}{J_{2}(p) \tau_{3}}\left(q_{1}+\arctan \left(\frac{J_{1}(p)}{J_{2}(p)} \sqrt{\frac{J_{2}(p)}{\omega(p)}} \dot{q}_{1}+\sqrt{\frac{J_{2}(p)}{\omega(p)}} \dot{q}_{2}\right)\right)\right]+\omega(p) \frac{J_{2}(p)}{J_{1}(p)} \sin \left(q_{1}\right),
\end{aligned}
$$

где дифференцирование ведется по времени $t$.

Отметим, что варьируя величину $x_{3}(0)$, получаем разные законы управления, из которых можно выбрать наиболее приемлемый. Кроме того, вид управления (7) можно упростить, если выбрать $K_{2}$ и $\tau_{3}$ таким образом, что $K_{2}=\frac{1}{\tau_{3}}$.

Пример. В качестве примера рассмотрим модель маятника с маховиком, которая имеет такие параметры: $m=0,04$ кг, $L=0,1 \mathrm{м}, M_{M}=9,4$ кг, $J_{R}=0,24 \cdot 10^{-4}$ кг $\cdot \mathrm{M}^{2}, r=0,28 \mathrm{м}, R=0,3 \mathrm{м}$, 
где $r$ и $R$ - соответственно внутренний и внешний радиусы маховика, который близок к кольцу. Момент инерции маховика можно рассчитать по формуле $J_{m}=\frac{M_{M}\left(r^{2}+R^{2}\right)}{2}$, а момент инерции маятника - по формуле $J_{m}=\frac{m L^{2}}{3}$. Исходя из результатов роботы [2], параметры управления могут быть выбраны такими: $K_{1}=\frac{1}{\tau_{2}}=8, K_{2}=0,05, \tau_{3}=10^{-4}$. Отметим, что в контексте данной модели выбор параметров $K_{2}$ и $\tau_{3}$ таким образом, что $K_{2}=\frac{1}{\tau_{3}}$, является нецелесообразным, поскольку накладывает слишком жесткие требования на электромотор. Пусть начальное положение маятника - его нижнее положение равновесия. Выбрав $x_{3}(0)=-\frac{J_{1}(p)}{J_{2}(p)} K_{1} \pi$ убедимся, что управление $\Delta$ стабилизирует верхнее положение равновесия маятника. Поведение модели иллюстрирует рис. 2.

Таким образом, в работе получен явный вид управления вращением маховика, предложенного в работе [2], которое обеспечивает стабилизацию верхнего положения равновесия маятника вне зависимости от начальных значений переменных, описывающих поведение модели. Явный вид управления, т. е. его зависимость от физических характеристик модели (скоростей, смещений, времени), является значимым для его практической реализации. Приемлемость полученного закона управления проиллюстрирована на примере реальной модели.

\section{ЦИТИРОВАННАЯ ЛИТЕРАТУРА}

1. Liu Y., Yu H. A survey of underactuated mechanical systems. IET Control Theory Appl. 2013. 7, Iss. 7. P. 92935. doi:https://doi.org/10.1049/iet-cta.2012.0505

2. Хорошун А.С. О стабилизации верхнего положения равновесия маятника вращением инерциального маховика. Прикл. механика. 2016. 52, № 5. С. 125-137.

3. Spong M.W., Corke P., Lozano R. Nonlinear control of the inertia wheel pendulum. Automatica. 2001. P 18451851. doi:https://doi.org/10.1016/S0005-1098(01)00145-5

4. Формальский А.М. Управление движением неустойчивых объектов. Москва: ФИЗМАТЛИТ, 2013. 235 с.

5. Quaiser Nadeem, Iqbal N., Hussain A., Qaiser Naeem Exponential stabilization of the inertia wheel pendulum using dynamic surface control. J. Circuits, Systems and Computers. 2007. 16, № 1. P. 81-92. doi:https://doi. org/10.1142/S0218126607003514

6. Reza Olfati-Saber Nonlinear control of underactuated mechanical systems with application to robotics and aerospace vehicles: Ph.D. thesis/ Massachusetts Institute of Technology. Cambridge, MA, 2001.

7. Song B., Hedrick J.K. Dynamic surface control of uncertain nonlinear systems. An LMI approach. London: Springer, 2011. $268 \mathrm{p}$.

Поступило в редакцию 20.11.2017

\section{REFERENCES}

1. Liu, Y. \& Yu, H. (2013). A survey of underactuated mechanical systems. IET Control Theory Appl., 7, Iss. 7, pp. 921-935. doi:https://doi.org/10.1049/iet-cta.2012.0505

2. Khoroshun, A. S. (2016). Stabilization of the Upper Equilibrium Position of a Pendulum by Spinning an Inertial Flywheel. Int.Appl.Mech., 52, Iss. 5, pp. 547-556. doi:https://doi.org/10.1007/s10778-016-0775-1 (in Russian). 
3. Spong, M. W., Corke, P. \& Lozano, R. (2001). Nonlinear control of the inertia wheel pendulum. Automatica., 37, pp. 1845-1851. doi:https://doi.org/10.1016/S0005-1098(01)00145-5

4. Formalsky, A. M. (2013). Motion Control of Unstable Objects. Moscow: FIZMATLIT (in Russian).

5. Quaiser, Nadeem, Iqbal, N., Hussain, A. \& Qaiser, Naeem (2007). Exponential stabilization of the inertia wheel pendulum using dynamic surface control. J. Circuits, Systems and Computers, 16, No. 1, pp. 81-92. doi:https:// doi.org/10.1142/S0218126607003514

6. Reza, Olfati-Saber (2001). Nonlinear control of underactuated mechanical systems with application to robotics and aerospace vehicles.(unpublished candidate thesis). Massachusetts Institute of Technology, Cambridge, MA.

7. Song, B. \& Hedrick, J.K. (2011). Dynamic surface control of uncertain nonlinear systems. An LMI approach. London: Springer.

Received 20.11.2017

\section{А.С. Хорошун}

Інститут механіки ім. С.П. Тимошенка НАН України, Київ

E-mail: khoroshunanatoliy@gmail.com

ПРО ПОБУДОВУ КЕРУВАННЯ РУХОМ МАЯТНИКА

ОБЕРТАННЯМ ІНЕРЦАЛЬНОГО МАХОВИКА

Розглянуто приклад малоприводної механічної системи, що являє собою маятник, керування рухом якого відбувається завдяки обертанню маховика. Отримано у явному вигляді закон керування обертанням маховика, який забезпечує стабілізацію верхнього положення рівноваги маятника.

Ключові слова: малоприводна механічна система, глобальна асимптотична стійкість, інерціальний маховик, положення рівноваги.

\section{A.S. Khoroshun}

S.P. Timoshenko Institute of Mechanics of the NAS of Ukraine, Kiev

E-mail: khoroshunanatoliy@gmail.com

\section{ON THE CONSTRUCTION OF A CONTROL OVER THE PENDULUM MOVEMENT BY THE ROTATION OF AN INERTIAL FLYWHEEL}

An example of the underactuated mechanical system, which is a pendulum, whose motion is controlled by the rotation of a flywheel, is investigated. The explicit form of the control law of the flywheel rotation, which ensures the stabilization of the upper equilibrium position of the pendulum, is obtained.

Keywords: underactuated mechanical system, global asymptotic stability, inertial flywheel, equilibrium position 